\title{
Characterizing the Response of Commercial and Industrial Facilities to Dynamic Pricing Signals from the Utility
}

\author{
${ }^{1}$ Johanna L. Mathieu, ${ }^{2}$ Ashok J. Gadgil, ${ }^{1}$ Duncan S. Callaway, \\ ${ }^{2}$ Phillip N. Price, ${ }^{2}$ Sila Kiliccote \\ ${ }^{1}$ University of California, Berkeley \\ Berkeley, CA, USA \\ ${ }^{2}$ Environmental Energy Technologies Division \\ Lawrence Berkeley National Laboratory \\ Berkeley, CA, USA
}

July 2010

This work was conducted at the University of California, Berkeley and Lawrence Berkeley National Laboratory under U.S. Department of Energy Contract No. DE-AC02-05CH11231. 


\title{
CHARACTERIZING THE RESPONSE OF COMMERCIAL AND INDUSTRIAL FACILITIES TO DYNAMIC PRICING SIGNALS FROM THE UTILITY
}

\author{
Johanna L. Mathieu \\ University of California, Berkeley \\ Berkeley, CA, USA \\ Ashok J. Gadgil \\ Lawrence Berkeley National Laboratory \\ Berkeley, CA, USA \\ Duncan S. Callaway \\ University of California, Berkeley \\ Berkeley, CA, USA \\ Phillip N. Price \\ Lawrence Berkeley National Laboratory \\ Berkeley, CA, USA \\ Sila Kiliccote \\ Lawrence Berkeley National Laboratory \\ Berkeley, CA, USA
}

\begin{abstract}
We describe a method to generate statistical models of electricity demand from Commercial and Industrial (C\&I) facilities including their response to dynamic pricing signals. Models are built with historical electricity demand data. A facility model is the sum of a baseline demand model and a residual demand model; the latter quantifies deviations from the baseline model due to dynamic pricing signals from the utility. Three regressionbased baseline computation methods were developed and analyzed. All methods performed similarly. To understand the diversity of facility responses to dynamic pricing signals, we have characterized the response of 44 C\&I facilities participating in a Demand Response (DR) program using dynamic pricing in California (Pacific Gas \& Electric's Critical Peak Pricing Program). In most cases, facilities shed load during DR events but there is significant heterogeneity in facility responses. Modeling facility response to dynamic price signals is beneficial to the Independent System Operator for scheduling supply to meet demand, to the utility for improving dynamic pricing programs, and to the customer for minimizing energy costs.
\end{abstract}

\section{INTRODUCTION}

Electricity markets are unusual in that electricity demand is relatively inelastic, in part because most electricity consumers do not monitor electricity prices. In addition, when generation nears its capacity supply also becomes increasingly inelastic. Traditionally, Independent Systems Operators (ISOs) balance electricity supply and demand by calling on the supply-side to produce electricity to meet demand. The result is extreme electricity price volatility on days when the load is high [1]. Several studies have attempted to understand and quantify the benefits of a more responsive demand-side [2-4].

One way of achieving Demand Response (DR) is through dynamic electricity pricing, defined here as any electricity pricing scheme in which prices are known to the customer no more than a day in advance. Per this definition, examples of dynamic pricing would include Critical Peak Pricing (CPP) and Real Time Pricing (RTP). Time of Use (TOU) pricing is not dynamic pricing under our definition.

Commercial and Industrial (C\&I) facilities using Energy Management Control Systems (EMCS) are likely candidates for dynamic pricing tariffs because responses to varying electricity prices can be automated. Responding to dynamic pricing includes both shedding and shifting electricity demand. Examples of load shedding strategies include changing Heating, Ventilating, and Air Conditioning (HVAC) set points and dimming/switching-off non- 
critical lighting. Examples of load shifting strategies include pre-cooling and water heating during times of the day when energy costs are low.

From the perspective of the ISO and the utility there is a strong need to accurately predict characteristics of C\&I facilities' demand sheds and shifts (including their variability) resulting from dynamic pricing signals. Better shed/shift forecasts are important to the ISO for scheduling electricity supply to meet demand. Predictions also allow the utility to understand how characteristics of dynamic pricing signal responses vary across facilities and which facilities are best suited to different DR programs. In addition, understanding facilities' responses to dynamic prices allows the utility to model, analyze, and compare new dynamic pricing programs and their ability to achieve the utility's goals (e.g., peak demand reductions, highly-predictable demand reductions, energy savings, etc.).

It is also advantageous for C\&I facilities to be able to predict characteristics of their own demand sheds/shifts. Facility managers would like to minimize energy costs subject to available resources, constraints (e.g., occupant comfort), and dynamic pricing information. Understanding how a facility responds to pre-programmed dynamic pricing response strategies is essential for minimizing energy costs. For instance, data presented in this paper show that the time interval between receipt of a demand response signal and complete execution of a demand reduction (referred to as 'ramp time' in this paper), using an automated demand response system, varies from less than three minutes to more than two hours. Facility managers should consider the ramp time of their facility when devising optimal price response strategies.

Another important reason to understand facility sheds/shifts resulting from dynamic pricing signals is to determine the optimal lead-time (defined here as the time between when prices are published and when they take effect) at which dynamic prices should be announced to the customer. It is advantageous to the facility to receive pricing information with large lead-times so that the facility can plan its response, leveraging all of its DR resources, to minimize total energy costs. However, to participate in real-time electricity markets, facilities would receive pricing information at short lead times (e.g., one hour), which would not allow them to leverage all of their DR resources (e.g., pre-cooling) resulting in smaller demand sheds. There are clearly tradeoffs in facility response to dynamic pricing as pricing information lead-time changes. Though this is not the subject of this paper, we are currently researching these tradeoffs.

One way to predict demand sheds and shifts, and their variability, is by modeling C\&I facilities. Facility models can be built with physical equations or historical electricity demand data. Existing physical equation-based facility models can accurately predict certain facility loads (e.g., HVAC loads, some lighting loads) if the characteristics of the facility are known and the model is calibrated with actual demand data. However, physical equation-based models seldom accurately predict human-controlled loads (e.g., some lighting loads, humancontrolled process loads, plug loads, etc.) and their variability. Alternatively, statistical models (which we employ in this paper) built with historical electricity demand data capture all facility loads and do not require calibration or knowledge of facility characteristics. However, historical electricity demand data are not generally sub-metered so different types of loads cannot be disaggregated. Also, statistical models capture only historical facility behavior and cannot be used to predict future facility behavior (though statistical models can be updated as more data become available).

In this paper, we describe a method to generate statistical models of C\&I facilities including their response to dynamic pricing signals. Facility models comprise a baseline demand model and a residual demand model that predicts deviations from the baseline model due to dynamic pricing signals. To understand the diversity of facility responses to dynamic pricing signals, we have characterized the response of 44 C\&I facilities participating in Pacific Gas and Electric's (PG\&E's) CPP Program.

We begin with a brief review of relevant research on DR. We then detail our approach and describe the data used in our analysis. We present our preliminary results and conclude.

\section{DEMAND RESPONSE}

DR is defined by DOE as "a tariff or program established to motivate changes in electric use by end-use customers in response to changes in the price of electricity over time, or to give incentive payments designed to induce lower electricity use at times of high market prices or when grid reliability is jeopardized" [3].

DR has many benefits. It can be used to reduce price volatility during peak periods [4]. It also contributes to system reliability [5]. In addition, DR could be used to provide ancillary services (A/S) such as spinning reserve [6] and regulation/load following [7]. 


\section{Participation Incentives}

There are many ways to achieve DR. For instance, in Interruptible Load Management (ILM) and Direct Load Control (DLC) programs customers allow the program sponsor (e.g., utility, aggregator, etc.) to control their loads within some prior agreed-upon constraints in exchange for credits and/or incentive payments. Much work as been done to develop strategies to control loads through ILM and DLC for peak load management [8-10].

DR can also be achieved through dynamic electricity pricing such as RTP and CPP. Many economists advocate RTP tied directly to wholesale prices $[1,4,11]$. Dynamic prices could also be a function of the "value and cost of electricity in different time periods" [3], which implies that they could encapsulate information other than wholesale prices. For instance, retail electricity prices could be higher during peak times (e.g., two times the wholesale price) to encourage power use reductions.

Another way to achieve DR is to allow loads to participate in wholesale electricity markets. For example, in the Participating Load Pilot (PLP) program conducted by PG\&E in California, C\&I facilities submit offers (their expected electricity load and a possible load reduction) and bids (price asked for achieving their offer) to the dayahead non-spinning reserve A/S market [12]. These bids/offers are optimized by the California ISO (CAISO) together with supply-side bids/offers, and if a facility's bid is accepted and they are called to act they must deliver their offered load reduction within minutes. While this particular program is not the subject of this paper, some data from this program are analyzed here.

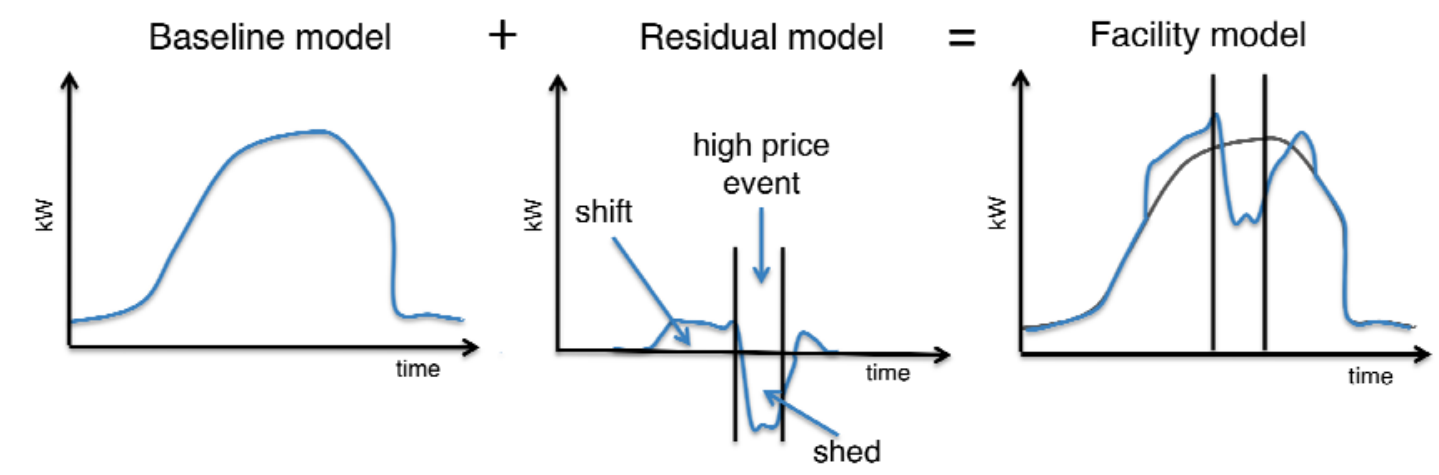

Figure 1. Illustration of how a baseline model and a residual model add to create a model of a facility's electricity demand.

\section{DR Strategies}

If a C\&I facility chooses to participate in a DR program it must develop price response strategies. Motegi et al. [13] present a number of commercial building control strategies for DR including HVAC strategies (global temperature adjustment of zones; adjustments to the air distribution and/or cooling systems), lighting strategies (switching off selected lighting zones, fixtures, and/or lamps; stepped dimming and/or continuous dimming), and turning off miscellaneous equipment. Other strategies for C\&I facilities could include shifting energy use to other times of the day (e.g., pre-cooling building thermal mass, shifting industrial processes), energy storage other than in the building thermal mass, and on-site generation.

A C\&I facility's DR strategies are limited by the facility's available resources and its control system's capabilities. Therefore, devices that are already automated such as HVAC components are commonly used in DR strategies.

\section{APPROACH}

A simple way to model a facility capable of responding to dynamic prices is to break the model into two parts: (1) a baseline model that predicts facility electricity demand on 'normal' days (i.e., days on which there is no dynamic pricing), and (2) a dynamic pricing response model that predicts changes in electricity demand (from the baseline) that result from dynamic pricing signals. This second model, referred to as a DR residual model in this paper, predicts both sheds in demand and shifts in energy use. Figure 1 illustrates how a baseline model and a residual model sum to create a model of a facility's electricity demand on a day in which there is one high price DR event that is known day-ahead (hence the ability of the facility to shift energy consumption to the period directly before the event). 
To create statistical models of facilities from historical electricity demand data, we performed the following tasks:

1. Developed piecewise linear and continuous baseline models (based on outdoor air temperature and 15minute interval demand data) using data from normal days.

2. Used the baseline model to predict what demand would have been on DR days and computed residuals between actual DR day data and baseline predictions.

3. Developed a list of parameters to characterize features of DR residuals (called 'residual parameters'). Examples include average demand shed, shed variability, and residual ramp time.

4. Computed DR residual parameters for all facilities for which data are available, and developed histograms for each parameter to inform a residual model.

\section{DATA SOURCES}

\section{Critical Peak Pricing (CPP) Demand Data}

The Critical Peak Pricing (CPP) Program is one of several PG\&E DR programs for large customers $(>200 \mathrm{~kW})$. The program incentivizes demand reductions, on up to twelve summer days per year (called 'DR days' in this paper), through a special tariff structure. In exchange for lower partial-peak and on-peak energy prices on non-DR days, energy prices on DR days are raised to three times the normal partial-peak rate from 12-3pm (moderate price period) and five times the on-peak rate from 3-6pm (high price period). DR days are announced by $3 \mathrm{pm}$ the business day before the event.

Fifteen-minute interval demand data from 44 C\&I facilities participating in the CPP Program were obtained from PG\&E and used to develop the histograms presented in this paper. All 44 facilities use Open Automation Demand Response Communication Standards (OpenADR), a software/hardware system that allows facilities to process DR signals or changing energy prices and to respond using pre-programmed control strategies [14]. OpenADR deployment began in 2006 [15,16], and so for each facility we have one to four years of demand data (2006-2009) depending upon when the facility began to participate in the CPP Program with OpenADR.

Currently, most of the 44 C\&I facilities considered in this paper use automated HVAC strategies to shed load. Several of the commercial buildings also use automated lighting strategies. The industrial facilities often use their automation system to shift or shed processes. Since the facilities receive day-ahead event notification, occupants who are told of the event will sometimes enact manual DR strategies such as pre-cooling the air in their office before the event or shutting down unused computers during the event. Currently, only one site has an automated strategy for pre-cooling and none of the sites have energy storage (besides in the buildings' thermal mass) or on-site generation that they can leverage specifically for DR events (though several of the sites have solar PV).

Unfortunately, none of these facilities' DR strategies are optimized to minimize ramp times. Therefore, a limitation to the analysis presented in this paper is that we are only able to characterize current behavior instead of the facilities' real capabilities. Therefore, estimates of residual parameters such as residual ramp time should be considered conservative.

\section{Participating Load Pilot (PLP) Program Demand Data}

Three facilities that had previously participated in the CPP Program with OpenADR were selected to participate in the 2009 PG\&E PLP Program [12]. Each of these facilities had exhibited fast ramp times ( $<15$ minutes $)$ to CPP events. In this pilot program, the facilities participated in the day-ahead $\mathrm{A} / \mathrm{S}$ (non-spinning reserve) market by submitting day-ahead demand forecasts and demand savings bids to the CAISO. Then, demand savings bids were pooled with bids from other generation resources and an optimized mix of bids was accepted by CAISO for that day. When a facility's demand savings bid was accepted and the facility was called to act, it was expected to drop its demand by the bid amount within ten minutes. Each of the facilities received the event signal and executed their automated demand reduction strategies using OpenADR. The facilities were not told the expected length of time for which the bid would be accepted.

The response strategies and subsequent behavior of facilities participating in the PLP program are similar to those seen for facilities participating in the CPP program. For each of the three participating facilities participating in the PLP program, high-resolution (four-second interval) demand data were available from PG\&E. The foursecond interval data were used to determine the high-resolution residual ramp times (as compared to the granular residual ramp times computed from the 15-minute interval CPP program data) presented in this paper. 
Two of the facilities that participated in the PLP program were commercial buildings that used HVAC strategies to achieve demand reductions. One building used the same strategies it had previously used to participate in the CPP program. The other building used feedback control to ensure that it maintained its desired load during the event period. A third facility that participated in the PLP program was an industrial facility that also used the same strategy it had previously used to participate in the CPP program. Specifically, it would shed a process when called upon to act.

\section{Outdoor Air Temperature Data}

For each facility, outdoor air temperature data from the nearest National Oceanic and Atmospheric Administration (NOAA) weather station were obtained from the National Climatic Data Center [17]. The data were collected by the weather station on approximately an hourly basis, though not generally on the hour. Unfortunately, much of the temperature data are missing. We have interpolated the temperature data in order to assign an approximate temperature to every 15 -minute interval (to match the interval of the CPP demand data). The algorithm is a simple linear interpolation between the data points on either side of the desired point. When six or more hours of data are missing we do not employ the interpolation algorithm and instead leave the temperature vector blank for those hours. In some cases, when the temperature data for a given site were particularly spotty we have filled the holes with data from a backup weather station (another nearby NOAA weather station). Another option would have been to build a model that maps temperature data from a nearby site to the given site, as is done in the wind energy community [18]. For all of the outdoor air temperature data used in our analysis, error results from the fact that none of the NOAA weather stations were co-located with the facilities.

Other weather data such as humidity could be used to build baseline models. Since all of the facilities analyzed in this study are located in low-humidity climates, we did not include humidity in our models. However, it would be interesting to consider adding humidity, especially when analyzing of facilities in more humid climates.

\section{RESULTS}

\section{Baseline Models}

Predicting electricity use for an individual facility is difficult. Many have researched methods to forecast electricity demand at the facility level [19-21]. To calculate baseline electricity use, utilities use simple methods. For example, PG\&E estimated individual baselines by averaging the electric use profile of the hottest three business days out of the last ten business days (called the 3/10 baseline), and sometimes multiplying by a 'morning adjustment' factor (which biases the curve up or down based on demand levels before a DR event). Coughlin et al. [22] evaluated this and several other methods for baseline computation to determine the bias and accuracy of each method. No single method emerged as consistently having both low bias and high accuracy for all of the facilities analyzed (suggesting that different baseline computation methods could be best suited for different facilities). They also found that morning adjustment factors consistently improved demand predictions; however, parameterizing a morning adjustment factor with data from DR days to predict baseline demand on DR days would bias demand predictions to be high on days when a facility shifts energy use to the morning hours (pre-cooling, industrial process shifting) in anticipation a DR event that has been announced day-ahead. Therefore, morning adjustment has not been considered here.

For our research, we compared three regression methods for baseline computation. Baselines for the 44 facilities participating in the CPP and PLP programs were constructed using 15-minute interval demand data. Since all 2006-2009 CPP DR days were called on non-holiday weekdays in May through September, baseline models for facilities participating in CPP were constructed only with demand data from non-holiday weekdays in May through September. All PLP events occurred on non-holiday weekdays in August through October. Baseline models for facilities participating in PLP were constructed with demand data from non-holiday weekdays in May through October. We chose not to look at season-to-season and month-to-month variation in facilities' electrical demand to keep sample sizes large.

Facilities' demand profiles also change year-to-year due to equipment upgrades, changes in usage patterns, etc. To reduce the chance of creating regression models with data from before and after significant changes (e.g., HVAC upgrades, which are often done in shoulder seasons) only one year worth of data were used to create each baseline. Therefore, facilities for which we have several years' worth of data have a different baseline each year. In total, we have 99 facility-years of data (where 'facility-year' is defined as one year of data for one facility).

For each facility-year, baseline models were computed using data from $\sim 70 \%$ of the available days (defined as weekdays with the exception of holidays and DR days). The remaining $(\sim 30 \%)$ of available days were used for 
model validation (these days are referred to as "MV days'). To compare baseline computation methods, we predicted baselines for MV days and subtracted the predictions from actual demand data to produce "MV residuals." Then for each facility, we computed the average and standard deviation of the MV residuals for each baseline computation method.

One problem with using non-DR day data to compute baselines for DR days is that DR days are generally hotter than non-DR days. Therefore, the baseline models presented in this paper may not be as robust for DR day temperatures (high temperatures) as they are for non-DR day temperatures.

Modeling Temperature-Dependent Demand For each baseline model, we assume that there are two components of electricity demand: (1) demand that varies by time of day and (2) temperature-dependent demand. By considering how a facility's HVAC system functions, we can guess the shape of the facility's temperaturedependent demand profile, assuming electricity is required for heating only to run pumps and fans (i.e. not as the heat source) (Figure 2). When it is cold enough outside that heating is required, electricity use increases as outdoor temperature goes down (i.e. as heating loads increase). A dead-band exists at moderate temperatures when the facility is neither heated nor cooled. When the outdoor temperature is high enough that cooling is required, the facility uses more electricity as temperature goes up (i.e. cooling loads increase) until the temperature is so high that the AC cooling capacity cannot achieve the desired indoor temperature set point, at which point demand is at the maximum possible AC load. On most summer days in California, facilities are cooling or, in some cases, operating in the dead-band.

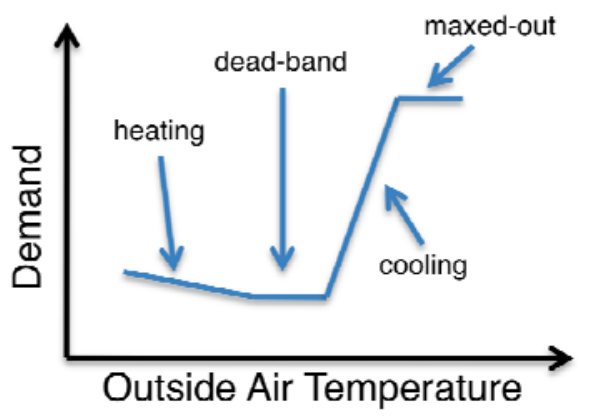

Figure 2. Illustration of the temperature-dependent demand profile of a facility, assuming no electric heating.

Baseline Method 1 Method 1 is similar in concept to Model 5 evaluated by Coughlin et al. [22]. In Method 1 , we perform a regression on outdoor air temperature, $T$, (the independent variable) and demand, $D$, (the dependent variable) at every 15 -minute interval, $i$, in a week, since we expect that electricity demand should be a function of the day of the week, time of the day, and outdoor air temperature. We assume facilities are usually operating in the cooling region and therefore we assume a linear temperature dependence. Demand, $D$, at time interval $i$, given $T(i)$ was computed as:

$$
D(i)=\alpha(i)+\beta(i) T(i)
$$

where $\alpha(i)$ approximates how load varies in time and $\beta(i)$ approximates how load varies as a function of temperature. Since time interval $i$ indicates a specific 15-minute interval in a week (e.g., Monday at 2:30pm, Wednesday at 8:45am), the index $i$ recycles every week; there are as many values of $D(i)$ and $T(i)$ as there are weeks in the data set. We generate $\alpha(i)$ and $\beta(i)$ by regressing over all of these values.

Since temperature and time of day are correlated, the parameter $\alpha$ may capture some of the variation in load that is actually due to temperature, and $\beta$ may capture some of the variability in load that is actually due to time-of-daydependent processes like occupancy. This is a limitation of this model only if the objective is one of inference; since we are exclusively interested in prediction this is not a limitation of this model.

Method 1 generates a total of 960 parameters (two for every 15 minute interval from Monday-Friday). This method allows the slope of the temperature-dependent load, $\beta$, to change over time, which may not be the case. 
Also, it assumes $\beta$ is constant in any given time interval, which is unlikely given Figure 2. The following two baseline methods address these issues.

Baseline Method 2 In Method 2 we chose to use six temperature parameters, $\delta_{j}$, each assigned to a different outdoor air temperature interval, to create a piecewise linear and continuous temperature-dependant demand model. While the temperature-dependent demand for each facility is not forced to look like that in Figure 2, the piecewise linear model used in Method 2 allows this sort of behavior, where Method 1 did not. Parameters were fit to data from time intervals when the facility was not operating in night mode since one would expect the facilities' response to temperature would change at night. ${ }^{1}$ The start and end of the night mode is selected manually by looking at average demand profiles on non-DR days.

For each baseline computation, temperature intervals assigned to each parameter are selected to ensure that the first and last temperature interval (e.g., $<50^{\circ} \mathrm{F}$ and $>90^{\circ} \mathrm{F}$ ) contain sufficient data for regression. The remaining temperature intervals are equally spaced between the first and last temperature intervals. Let $B_{k}$ for $k=1 \ldots 5$ be the bounds of the temperature intervals (e.g., $50^{\circ} \mathrm{F}, 60^{\circ} \mathrm{F}, 70^{\circ} \mathrm{F}, 80^{\circ} \mathrm{F}$, and $90^{\circ} \mathrm{F}$ ). To achieve piecewise linearity and continuity, at each time interval, $i$, the outside air temperature, $T(i)$, is broken into six component temperatures, $T_{c}(i, j)$, one of which is assigned to each temperature parameter. Component temperatures are computed as follows:

1. If $T(i)>B_{1}$ then $T_{c}(i, 1)=B_{1}$. Otherwise, $T_{c}(i, 1)=T(i)$ and $T_{c}(i, m)=0$ for $m=2 \ldots 6$ and algorithm is ended.

2. For $n=2 \ldots 4$, if $T(i)>B_{n}$ then $T_{c}(i, n)=B_{n}-B_{n-1}$. Otherwise, $T_{c}(i, n)=T(i)-B_{n-1}$ and $T_{c}(i, m)=0$ for $m=(n+1) \ldots 6$ and algorithm is ended.

3. If $T(i)>B_{5}$ then $T_{c}(i, 5)=B_{5}-B_{4}$ and $T_{c}(i, 6)=T(i)-B_{5}$.

Each $T_{c}(i, j)$ is multiplied by its $\delta_{j}$ and then summed to determine the temperature-dependent load.

Daytime demand, $D_{d}$, at time interval $i$, is computed as follows:

$$
D_{d}(i)=\alpha(i)+\sum_{j=1}^{6} \delta_{j} T_{c}(i, j)
$$

As in Method 1, $\alpha(i)$ characterizes the temperature-independent load.

To predict nighttime demand, $D_{n}$, a single temperature parameter, $\delta_{n}$, is fit to data from intervals when the facility is in night mode:

$$
D_{n}(i)=\alpha(i)+\delta_{n} T(i)
$$

Method 2 generates a total of 487 parameters (one for every 15 minute interval from Monday-Friday, six daytime temperature parameters, and one nighttime temperature parameter).

Baseline Method 3 Method 3 similar to Method 2; however, it takes into account not only outdoor air temperature but also outdoor air temperature trend. An additional parameter, $\eta$, captures the temperature trend during the non-night modes of facility operation. The temperature trend is defined as the temperature in the current interval, $T(i)$, minus the average temperature over the past three hours, $\bar{T}_{3 h r s}(i)$. The daytime demand is predicted with the equation:

$$
D_{d}(i)=\alpha(i)+\sum_{j=1}^{6} \delta_{j} T_{c}(i, j)+\eta\left(T(i)-\bar{T}_{3 h r s}(i)\right)
$$

Nighttime demand, $D_{n}$, is calculated as in equation (3).

Method 3 generates a total of 488 parameters (one for every 15 minute interval from Monday-Friday, six daytime temperature parameters, one nighttime temperature parameter, and one temperature trend parameter).

\footnotetext{
1 It is likely that a facilities' response to temperature would be different in each of its operating modes (e.g., night, normal, warm-up, cool-down, etc.). Ideally, one would determine separate temperature parameters for each of these modes. Unfortunately, we do not have information about when buildings switch modes and it is not obvious from the data (except when buildings go from night to warm-up mode and cool-down to night mode). Therefore, we have not attempted to distinguish between other operating modes.
} 
Comparison of Baselines Example plots of average non-DR demand and average model validation residual ("MV residual") for one facility participating in the CPP program are given in Figure 3, subplots A, and B, respectively. In both subplots, dotted lines show one standard deviation from the mean. In subplot A, the vertical lines indicate our differentiation between daytime and nighttime demand (used in Method 2 and 3).

Figure 4 gives histograms of certain MV residual parameters (average daily MV residual power, maximum daily MV residual power, and average of the daily standard deviation of MV residual power) computed for an average non-DR day, for each of the 99 facility-years, and with each of the baseline methods (M1 is Method 1, etc.). To compare results between facility-years, power values were normalized by dividing by the maximum demand on an average non-DR day for each facility-year. Ideally, each MV residual parameter would be zero. Daily MV residual parameters were surprisingly consistent between baseline methods and these histograms show that no baseline method consistently performed better than the rest. In the future, it would be worth analyzing MV residual parameters for specific hours of the day to determine if the accuracy of the three baseline computation methods varies throughout the day.
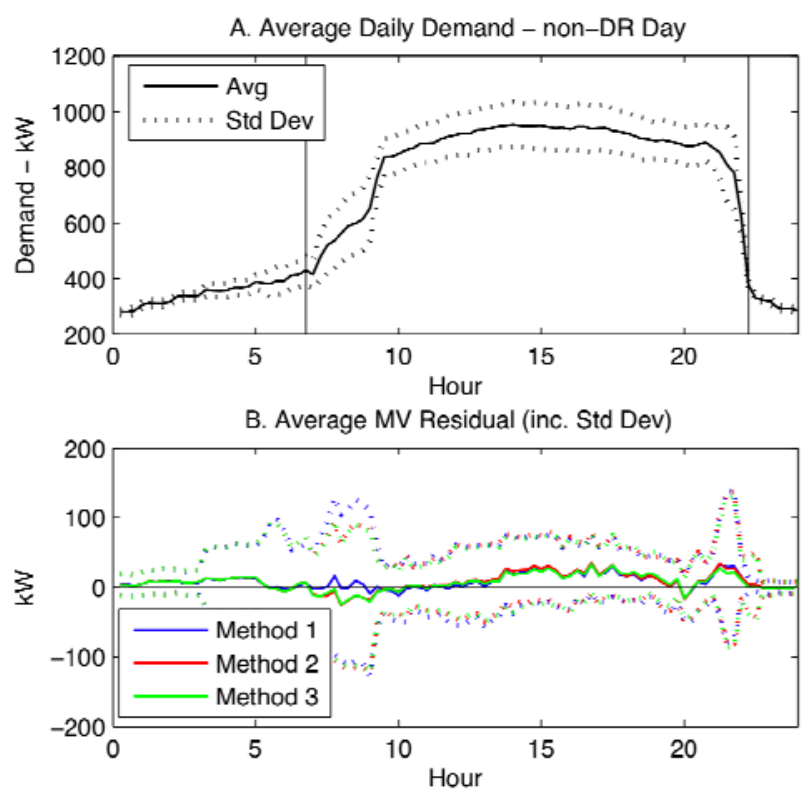

Figure 3. (A) Daily demand and (B) MV residuals for one commercial facility. 

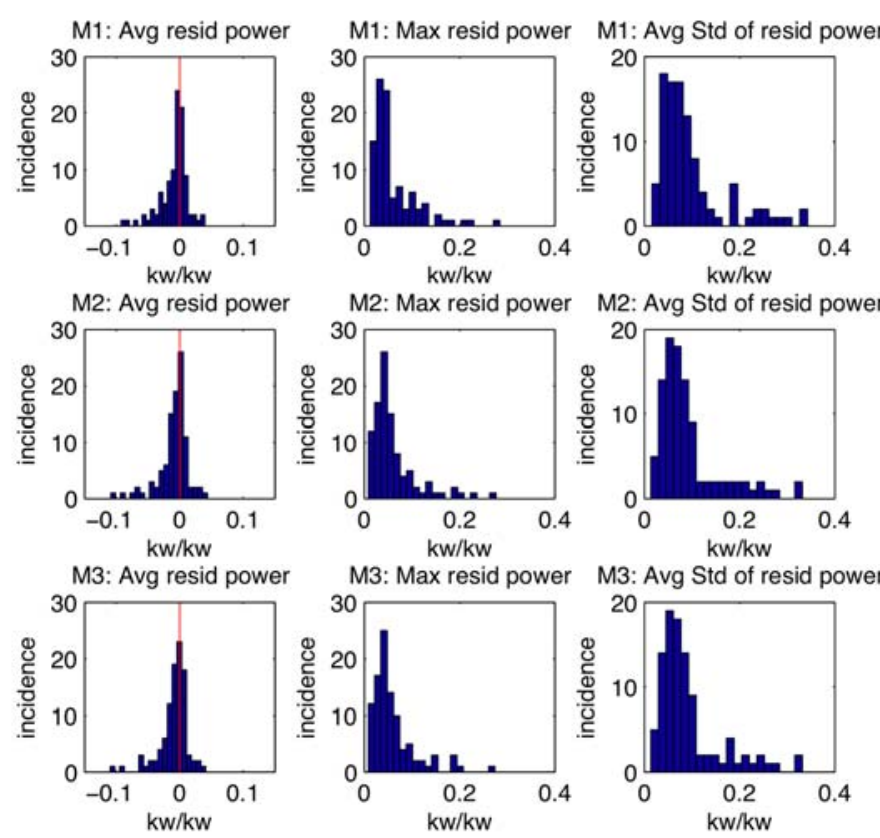

Figure 4. MV residual parameter histograms comparing three baseline methods.

All baseline computation methods seem to exhibit relatively low bias (i.e., for $\sim 99 \%$ of facilities average residual power is within $\pm 10 \%$ of the maximum demand on average non-DR day for that facility). Also, for $\sim 97 \%$ of the facilities the maximum residual power is within $\pm 20 \%$ of the facility's maximum demand on average non-DR day. It is important to note that MV residuals appear auto-correlated and we have not yet addressed this issue. Given our existing information, the best choice of baseline is Method 2 since is able to predict electricity demand seemingly as well as the other two methods while using less parameters.

\section{DR Residuals}

All three baseline methods were used to predict baseline demand on DR days (both CPP and PLP DR days) for all 44 facilities. DR day residuals were computed by subtracting baseline predictions from actual demand data.

For each facility participating in the CPP program, we computed the average and standard deviation of the DR residuals for each baseline computation method. Example plots including average baselines plotted on top of average DR day demand data and average DR residuals for one facility participating in the CPP program (the same as the facility as in Figure 3) are given in Figure 5, subplots A and B, respectively. In subplot B, the dotted lines show one standard deviation from the mean. In both plots, the vertical lines indicate the CPP starting, middle, and ending times. 

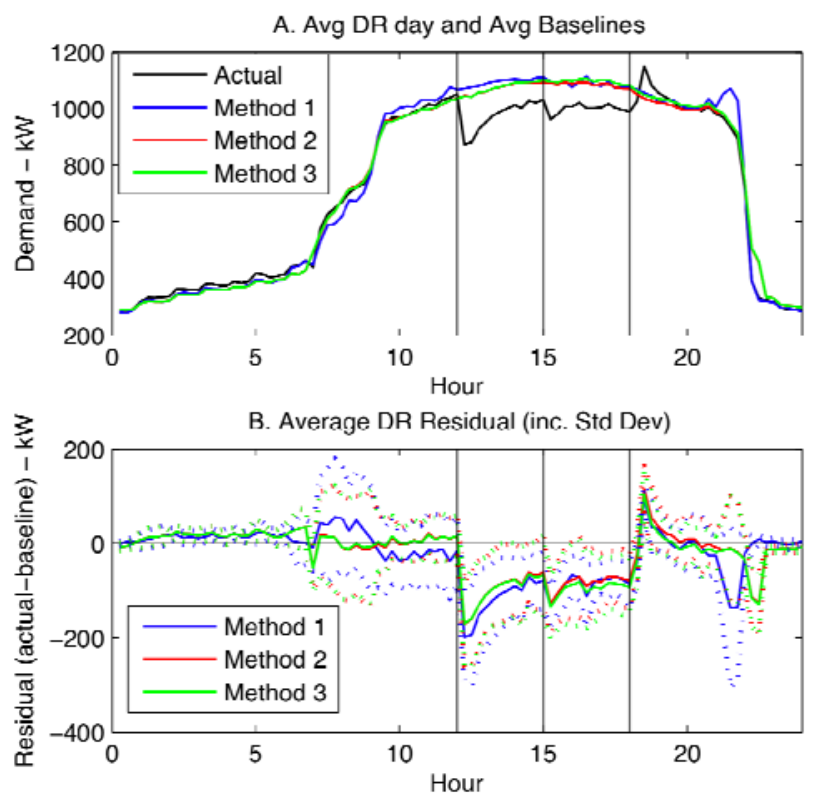

Figure 5. (A) Baselines and (B) DR residuals for one commercial facility participating in the CPP program.

To compute PLP event DR residuals, we interpolated 15-minute interval baseline predictions to create foursecond interval baseline predictions and then subtracted these baseline predictions from the actual four-second demand data. DR residuals from facilities participating in the PLP program cannot be averaged because events happen at different times throughout the day.

\section{Parameters used to Characterize DR Residuals}

The purpose of defining and quantifying residual parameters is to inform the residual model. In general, it is better to determine DR characteristics from DR residuals rather than from the data for actual DR day because residuals are corrected for demand changes due to time of week and temperature. We developed a list of parameters used to characterize DR residuals for facilities participating in the CPP Program.

- Average demand shed $(\mathrm{kW})$ : the average power shed during the DR event, calculated separately for the moderate and high price periods

- Shed variability (standard deviation of demand shed) $(\mathrm{kW}$ ): the standard deviation of power shed during the DR event, calculated separately for both the moderate and the high price periods

- Residual ramp time (min): the amount of time for demand to drop to the average demand shed during the DR event, calculated separately for both the moderate and high price periods.

- Energy saved $(\mathrm{kWh})$ : the difference between the baseline predicted energy use and the actual energy use, calculated separately for both the moderate and high price periods

- Energy shifted (morning) ( $\mathrm{kWh}$ ): the difference between the baseline predicted energy use and the actual energy use in the morning, calculated for the period between the beginning of the warm-up mode (chosen manually) and the start of the DR event ${ }^{2}$

- Energy recovered (evening) ( $\mathrm{kWh}$ ): the difference between the baseline predicted energy use and the actual energy use in the evening, calculated for the period between the end of the DR event and the beginning of the night mode (chosen manually)

- Maximum rebound peak $(\mathrm{kW}):^{3}$ the maximum difference between the baseline predicted power and the actual power consumed between the end of the DR event and the beginning of the night mode (chosen manually)

\footnotetext{
2 In some cases, the facility exhibits a strong start-up peak (usually due to cooling of the facility after a hot night). If this start-up peak was observed in the DR residual (i.e. it was not predicted by the baseline methods), then shifted energy is calculated for period between the end of the start-up peak (chosen manually) and the start of the DR event. Otherwise, values for shifted energy would have been biased (high).
} 
- Percent Average Power (whole day) (\%): the actual average daily demand divided by the baseline-predicted average daily demand

- Percent Maximum Power (whole day) (\%): the actual maximum daily demand divided by the baseline-predicted maximum daily demand

- Percent Energy Used (whole day) (\%): the actual daily energy use divided by the baseline-predicted daily energy use

Other DR residual parameters could be defined to understand other aspects of the DR residuals. Examples include morning power peak $(\mathrm{kW})$, morning ramp rate $(\mathrm{kW} / \mathrm{hr})$, and demand shed as a function of temperature.

In order to compare results between facility-years, parameters with units of power $(\mathrm{kW})$ were normalized with the maximum demand $(\mathrm{kW})$ on an average DR day for that facility-year, predicted by the baseline method. Parameters with units of energy $(\mathrm{kWh})$ were normalized with the energy consumed on an average DR day for that facility-year, predicted by the baseline method.

Since PLP events happen at different times throughout the day and for different durations, high-resolution residual ramp time was the only parameter used to characterize PLP DR residuals. High-resolution residual ramp time is defined in the same way as residual ramp time - the amount of time for demand to drop to the average demand shed during the PLP event. For short PLP events, facilities might not have enough time to complete their response; for this reason we defined "short" PLP events as those lasting less than 15 minutes and did not consider them in the analysis. On days when more than one PLP event was called we have only considered the first PLP event of the day since subsequent events were often called while the facility was still recovering from the first event.

It is important to note that all DR residual parameters may be correlated and it will be important to take into account any cross-correlations when creating residual models based on these parameters.

\section{Histograms for each Parameter from CPP Data}

Values for the residual parameters defined in the previous subsection were calculated for facilities participating in the CPP program, for each facility-year available, using average DR residuals and baseline computation Method 2. After parameters were normalized, histograms were generated for each parameter (Figure 6). Note that 'Percent Average Power' has the same histogram as 'Percent Energy Used' and therefore the histogram for 'Percent Average Power' is not included in Figure 6. The purpose of histograms is to understand the distribution of residual parameters seen in the 99 facility-years of data. In Figure 6, red vertical lines mark values that would be expected if the facilities were behaving exactly as predicted by the baseline (these values are 0 or 100 , depending upon the parameter definition). Note that in the residual ramp time histograms each bar represents a 15 -minute interval (e.g., the fifth bar shows facilities with ramp times between 60 and 75 minutes).

Since only 15-minute interval demand data were available for facilities participating in the CPP program, time response results are granular. The histograms show that most facilities respond to dynamic prices within 15 minutes of the start of the event; however, some facilities take over two hours to respond. This result is partially a function of the way in which the residual ramp time is defined. For instance, if a facility has a strategy whereby they respond within 15 minutes but then shed a much larger load half way through the DR period (i.e. 90 minutes into the event), the calculated residual ramp time would be larger than 15 minutes.

Moreover, facility response to dynamic pricing signals is not instantaneous because of many real factors such as delays in facility sequence of controls, device cool-down periods, slow telemetry, lagging manual DR strategies, illtimed control systems, etc. Ramp times are a function of the types of loads a facility sheds. For instance, we would expect that shedding lighting loads would be nearly instantaneous while shedding HVAC loads may take some time. The facility-level demand data we have used in our analysis have only allowed us to compute each facility's total system residual ramp time; however, it would be advantageous to understand the residual ramp time of each of the facility's subsystems.

\footnotetext{
${ }^{3}$ Many facilities try to ensure that they do not generate new demand peaks directly after the event since facilities are charged for peaks in addition to their energy consumption. Therefore, most energy and power peaks in the recovery period (referring to the period immediately after the DR event) are lower than if facilities had simply reverted back to pre-event HVAC set points after the event.
} 
The residual histograms show that most facilities reduce power use and save energy during DR events (unsurprising); however, the standard deviation of the demand shed is often large. In some cases the demand shed is negative, which means that the facility's power use increases during the DR event. This could be because (1)
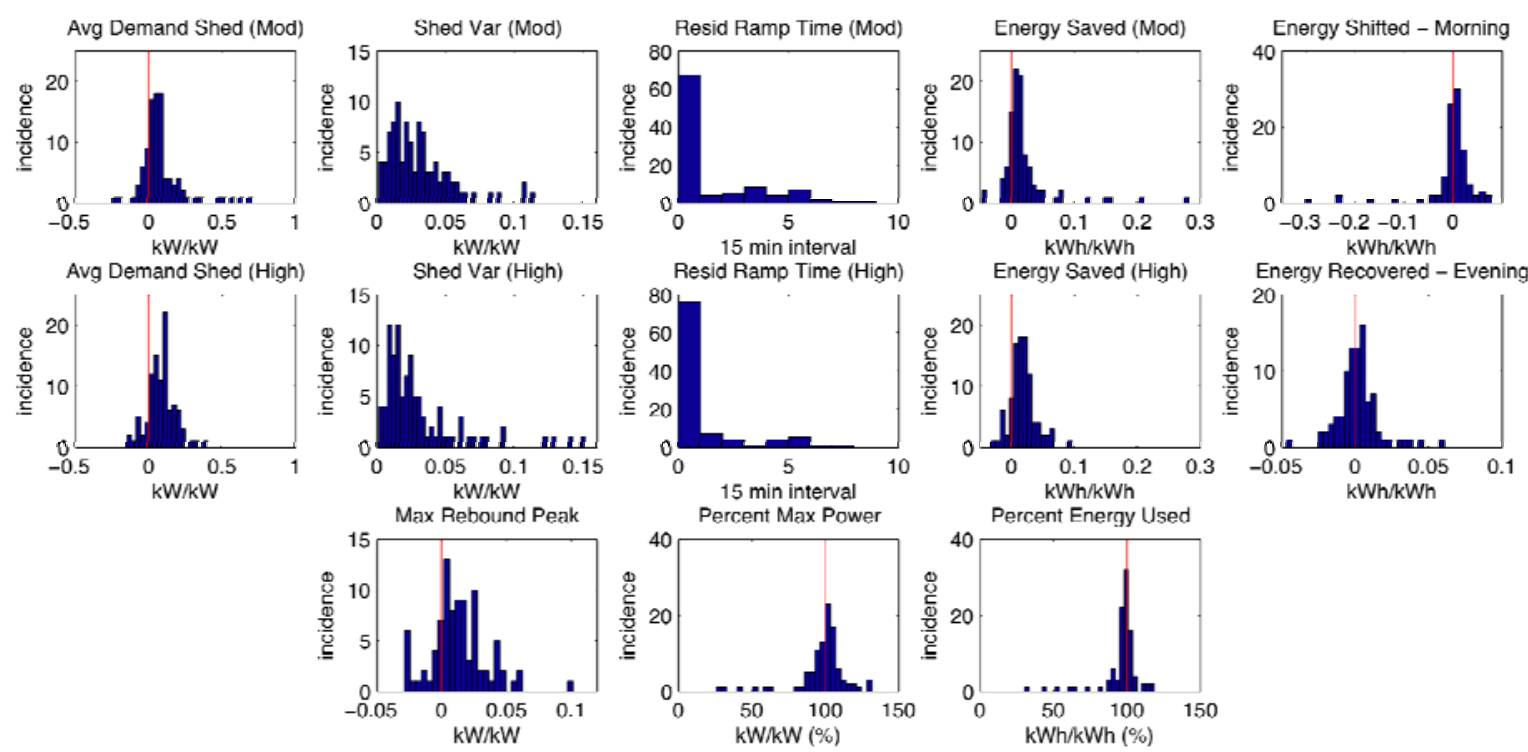

Figure 6. DR residual parameter histograms ('Mod' = CPP Moderate Price Period, 'High' = CPP High Price Period).

despite automation, the facility is not responding to DR events, or (2) the baseline model is inaccurate. While some facilities shift energy use to the morning others actually use less than predicted in the morning indicating that there is no clear trend to shift energy

use to the morning among facilities in the data set. The same holds for energy recovery. Most facilities exhibit a slight rebound peak after the DR event; however several of the facilities make it into night mode without peaking.

Results also show that average power use and energy use decrease for only a little more than half of the facilities as a result of the DR program. The maximum daily power use actually goes up for most facilities participating in the DR program. This means that the program does not necessarily lower the facilities' demand peaks but rather moves them to different parts of the day.

Autocorrelation and error resulting from our limited data set will need to be formally dealt with before we are able to characterize the statistical significance of our results. For this reason we only present our results in the histograms in Figure 6 (i.e. we do not show tables with average shed characteristics and confidence intervals).

\section{High-Resolution Residual Ramp Time from PLP Data}

Since residual ramp times computed from CPP data were granular, high-resolution (four-second interval) PLP data were used to compute high-resolution residual ramp times for the three facilities participating in the PLP program. Since demand data from PLP event days cannot be averaged (PLP events happen at different times and for different durations), high-resolution residual ramp times were computed for each PLP event for each facility participating in the PLP program. Table 1 gives the mean, minimum, and maximum residual ramp time for each facility. Facilities participated in six to seven PLP events during the summer of 2009 and only a small number of those events met our criteria (lasted longer than 15 minutes and was the first event of the day). The number of events from which the ramp times have been computed is listed in the table.

Table 1. High-resolution residual ramp times computed for three facilities participating in the PLP program.

\begin{tabular}{llllll}
\hline & $\begin{array}{l}\text { Type of } \\
\text { Facility }\end{array}$ & Events & $\begin{array}{l}\text { Mean } \\
(\mathrm{min})\end{array}$ & $\begin{array}{l}\text { Min } \\
(\mathrm{min})\end{array}$ & $\begin{array}{l}\text { Max } \\
(\mathrm{min})\end{array}$ \\
\hline Facility 1 & Commercial & 4 & 7.9 & 2.7 & 16.7 \\
Facility 2 & Commercial & 3 & 2.7 & 1.6 & 3.8
\end{tabular}


\begin{tabular}{llllll} 
Facility 3 & Industrial & 2 & 3.8 & 1.8 & 6.0 \\
\hline
\end{tabular}

\section{CONCLUSIONS}

We have described a method to generate statistical models of electricity demand from C\&I facilities including their response to dynamic pricing signals using historical electricity demand data. Facility models are the sum of a regression-based baseline model and a residual model. To inform the residual model, DR residuals were parameterized and parameter values were computed for 99 facility-years of electricity demand data from C\&I facilities that have participated in PG\&E'S CPP Program. Parameters were plotted on histograms to understand the diversity of facility responses to dynamic pricing signals. In most cases, facilities shed load during demand response events but there is significant heterogeneity in their responses including some cases in which facilities exhibit a negative demand shed. Also, despite automation, there is a diversity of residual ramp times.

In the future, this information can be used to build a model of a hypothetical community of facilities (e.g., a virtual test bed of 10,000 facilities), reflecting the actual diversity of responses to dynamic price signals from a California utility. As more data become available this aggregated model can be updated to reflect current facility behavior. Since many of the residual parameters defined in this paper are likely correlated, the aggregated model must take into account cross-correlation. Moreover, autocorrelation in model error should be addressed.

\section{ACKNOWLEDGMENTS}

Thanks to Mary Ann Piette and Alex Bayen for their helpful comments. Also, thanks to PG\&E for the CPP and PLP data, and the LBNL Demand Response Research Center for facilitating access it. Johanna Mathieu is funded by a UC Berkeley Chancellor's Fellowship.

\section{REFERENCES}

[1] Borenstein, S., 2002, "The Trouble With Electricity Markets: Understanding California's Restructuring Disaster," J. Economic Perspectives, 16, pp. 191-211.

[2] FERC, 2009, "A National Assessment of Demand Response Potential," www.ferc.gov/legal/staff-reports/06-09demand-response.pdf

[3] DOE, 2006. "Benefits of Demand Response in Electricity Markets and Recommendations for Achieving Them," US Department of Energy report to the US Congress, http://www.oe.energy.gov/DocumentsandMedia/congress_1252d.pdf.

[4] Borenstein, S., M. Jaske, and A. Rosenfeld, 2002, "Dynamic Pricing, Advanced Metering and Demand Response in Electricity Markets," University of California Energy Institute: Center for the Study of Energy Markets Paper CSEMWP-105.

[5] NERC, 2008, "2008 Long-Term Reliability Assessment: 2008-2017," North American Electric Reliability Corporation Report, www.nerc.com/files/LTRA2008.pdf.

[6] Eto, J., C. Goldman, G. Heffner, B. Kirby, J. Kueck, M. Kintner-Meyer, J. Dagle, T. Mount, W. Schultze, R. Thomas, and R. Zimmerman, 2002, "Innovative Developments in Load as a Resource," Proc. of IEEE Power Engineering Society Winter Meeting, 2, pp. 1002-1004.

[7] Callaway, D.S., 2009, "Tapping the Energy Storage Potential in Electric Loads to Deliver Load Following and Regulation, with Application to Wind Energy," Energy Conversion and Management, 50, pp. 1389-1400.

[8] Burke, W. and D. Auslander, 2009, "Low Frequency Pulse Width Modulation Design for HVAC Compressors," Proc. ASME 2009 IDETC/CIE Conference, San Diego, CA.

[9] Huang, K., H. Chin, and Y. Huang, 2004, "A Model Reference Adaptive Control Strategy for Interruptible Load Management," IEEE Trans. on Power Systems, 19, pp. 683-689.

[10] Navid-Azarbaijani, N. and M. Banakar, 1996, "Realizing Load Reduction Functions by Aperiodic Switching of Load Groups," IEEE Trans on Power Systems, 11, pp. 721-727.

[11] Bushnell, J., B. Hobbs, and F. Wolak, 2009, "When it Comes to Demand Response is FERC is own Worst Enemy?" The Electricity Journal, 22(8), pp. 9-18.

[12] Kiliccote, S., M.A. Piette, G. Ghatikar, E. Koch, D. Hennage, J. Hernandez, A. Chiu, and J. Goodin, 2009, "Using Open Automated Demand Response Communications in Demand Response for Wholesale Ancillary Services," Proc. of Grid Interop Forum, Denver, CO.

[13] Motegi, N., M.A. Piette, D.S. Watson, S. Kiliccote, and P. Xu, 2007, "Introduction to Commercial Building Control Strategies and Techniques for Demand Response," Lawrence Berkeley National Laboratory Report LBNL-59975. 
[14] Piette, M.A., S. Kiliccote, and G. Ghatikar, 2008, "Linking Continuous Energy Management and Open Automated Demand Response," Proc. of Grid Interop Forum, Atlanta, GA.

[15] Piette, M.A., D. Watson, N. Motegi, and S. Kiliccote, 2007, “Automated Critical Peak Pricing Field Tests: 2006 Pilot Program Description and Results," Lawrence Berkeley National Laboratory Report LBNL-62218.

[16] Wikler, G., I. Bran, S. Prijyandonda, S. Yoshida, K. Smith, M.A. Piette, S. Kiliccote, G. Ghatikar, D. Hennage, and C. Thomas, 2007, "Pacific Gas \& Electric Company 2007 Auto-DR Program Task 13 Deliverable: AutoDR Assessment Study," http://drrc.lbl.gov/pubs/pge-auto-dr-assessment-study.pdf.

[17] NOAA, 2009, "NNDC Climatic Data Online," Satellite and Information Service National Climate Data Center, http://www7.ncdc.noaa.gov/CDO/dataproduct.

[18]Landberg, L., L. Myllerup, O. Rathmann, E.L. Petersen, B.H. Jørgensen, J. Badger, N.G. Mortensen, 2003, "Wind Resource Estimation - An Overview," Wind Energy, 6(3), pp. 261-271.

[19] Pedersen L., J. Stang, and R. Olsen, 2008, "Load Prediction Method for Heat and Electricity Demand for the Purpose of Planning for Mixed Energy Distribution Systems," Energy and Buildings, 40, pp.1124-1134.

[20] Karatasou S., M. Santamouris, and V. Geros, 2006, "Modeling and Predicting Building's Energy Use with Artificial Neural Networks: Methods and Results," Energy and Buildings, 38 pp. 949-958.

[21] Kreider J.F., and J.S. Haberl, 1994, "Predicting Hourly Building Energy Use: The Great Energy Predictor Shootout-Overview and Discussion of Results," ASHRAE Transactions, 100 pp. 1104-1118.

[22] Coughlin, K., M.A. Piette, C. Goldman, and S. Kiliccote, 2009, "Statistical Analysis of Baseline Load Models for Non-Residential Buildings," Energy and Buildings, 41, pp. 374-381. 